Bull. Mater. Sci., Vol. 30, No. 1, February 2007, pp. 51-56. () Indian Academy of Sciences.

\title{
Electrical conductivity of polyaniline doped PVC-PMMA polymer blends
}

\author{
S H DESHMUKH*, D K BURGHATE, $V$ P AKHARE, $V$ S DEOGAONKAR, \\ $P$ T DESHMUKH ${ }^{\dagger}$ and $M$ S DESHMUKH ${ }^{\dagger \dagger}$ \\ Department of Physics, Shri Shivaji Science College, Amravati 444 603, India \\ ${ }^{\dagger}$ Department of Chemistry, Dr Panjabrao Deshmukh Polytechnic, Amravati 444 603, India \\ ${ }^{\dagger}$ Department of Chemistry, Matoshri Vimlabai Deshmukh Mahavidyalaya, Amravati 444 603, India
}

MS received 27 June 2006; revised 7 August 2006

\begin{abstract}
The electrical conductivity of polyaniline doped polyvinylchloride (PVC) and poly(methyl methacrylate) (PMMA) thin films has been measured by studying the $I-V$ characteristics at various temperatures in the range 323-363 K. The results are presented in the form of $I-V$ characteristics and analysis has been made by interpretation of Poole-Frenkel, Fowler-Nordheim, Schottky $\ln (J)$ vs $T$ plots, Richardson and Arrhenius plots. The analysis of these results suggests that Schottky and Richardson mechanisms are primarily responsible for the observed conduction.
\end{abstract}

Keywords. Polyaniline doped PVC-PMMA polymer blends; conductivity.

\section{Introduction}

Polymer composites have steadily gained growing importance during the past decade. A good amount of work has been reported on the conduction mechanism in polymeric materials. The electrical conduction in polymer film has much importance due to the discovery of the memory phenomenon (Kryezewski 1975) and has wide applications now-a-days in thin film devices (Mead 1961). In recent years, because of the need for electrostatic charges dissipation, electromagnetic shielding etc, new polymers with electrical conductivity have been formulated. This led to new discoveries in the area of conducting polymers, which has now become one of the hot topics of research (Radhakrishnan 2001).

Electrical properties constitute one of the most convenient and sensitive methods for studying the polymer structure (Ferraro and Walkar 1965; Kimura and Kajiwara 1998). The interest in organic and polymeric semiconductors has arisen, particularly because of their electrophotographic and solar cell applications. Many synthetic polymers (Danno et al 1983; Kumar et al 1985) like polyacetylene, polypyrrole, poly-carbazol etc have been studied.

The electrical conduction in iodine doped polystyrene (PS) and poly(methyl methacrylate) (PMMA) has already been reported (Chakraborty et al 1991; Sangawar 1995). Keller et al (1991) reported the thermally stimulated discharge current (TSDC) study of polyblends of PS and PMMA. Belsare and Deogaonkar (1998) measured the elec-

*Author for correspondence trical conductivity of iodine doped polyblend films of polystyrene (PS) and poly(methyl methacrylate) (PMMA).

Electrical conduction of succinic acid doped glycine pellet has been discussed by Burghate et al (2004). Karimi et al (1987) carried out study of electrical conduction in polyethyleneterepthalate (PET) film. The electrical properties of regenerated cellulose (cello phone) film were studied by Bhat and Makwana (1990). Khare (1994) studied the current-voltage characteristics of malachite green doped cellulose acetate films, for the measurement of electrical conductivity, as function of temperature, field, thickness and dopant concentration, using a conventional sandwich type cell.

Deshmukh et al (2005) reported electrical conduction in semiconducting PVC-PMMA thin film. Microhardness and X-ray diffraction studies on polymer blends of polyethylene methacrylate (PEMA) and polyethyleneoxide (PEO) have been reported by Awasthi and Bajpai (2001).

In the case of organic solids, where the conductivity due to electron exiting from valence band to conduction band (Keton 1968; Khare and Shriwastava 1991) is negligible, a complex conduction behaviour (Parak and Garg 1991; Khare 1994) has been explained usually in terms of electron emission from cathode i.e. Schottky-Richardson mechanism (Schottky 1914), or the electron liberation from traps in the bulk of the material i.e. Poole-Frenkel mechanism (Frenkel 1938). However, the possibility of tunneling (Fowler and Nordheim 1928) and space charge limited conduction (Rose 1955) have also been investigated in the literature. 
The doping of iodine in PS (Chakraborty et al 1991), in PMMA (Sangawar 1995) and polyblends of PS and PMMA (Belsare and Deogaonkar 1998), have been reported to possess considerable influence on the conductivity although no attempt, so far, seems to have been made to study the effect of polyaniline conducting polymer doped blends. PVC as proton donor and PMMA as proton acceptor are selected for the present study. The choice of these polymers thus takes into account the donor-acceptor capabilities. In the present study, d.c.-conduction of donoracceptor mixed polymers doped with polyaniline, was measured to identify the mechanism of electrical conduction. It is shown how the $I-V$ data of the sample can be used to arrive at a possible conclusion. Results have been discussed in the light of different mechanisms, such as Poole-Frenkel, Fowler-Nordheim, $\ln (J)$ vs $T$ plots, Schottky plots, Richardson plots and Arrehenius plots. In case of SchottkyRichardson mechanism, the current shows strong temperature dependence but not in case of Poole-Frenkel mechanism. The study of temperature dependence of current density is, therefore, of great importance. The X-ray diffractogram (XRD) shows that the crystallinity is almost negligible showing the sample is amorphous.

\section{Experimental}

\subsection{Preparation of sample}

Polyvinylchloride (PVC) of standard grade supplied by Polychem Industries, Mumbai and poly(methyl methacrylate) (PMMA) supplied by Dental Product of India Ltd., Mumbai, were used for the study. The conducting polymer (polyaniline) was prepared by chemical oxidation using ferric chloride by conventional procedure. For the preparation of polyaniline doped thin film, the two polymers, PVC ( $1.5 \mathrm{~g})$ and PMMA $(0.5 \mathrm{~g})$, were taken in the ratio $3: 1$ by weight, $1.5 \mathrm{~g}$ of PVC in $15 \mathrm{ml}$ of tetrahydrofuran (THF) and $0.5 \mathrm{~g}$ of PMMA in $5 \mathrm{ml}$ of THF dissolved separately and subsequently mixed together. Polyaniline was taken in $2 \mathrm{wt} \%$ and was dissolved in $5 \mathrm{ml}$ of THF to produce polyaniline solution. After allowing them to dissolve completely, the three solutions were mixed together. The solution was heated at $60^{\circ} \mathrm{C}$ for $2 \mathrm{~h}$ to allow polymers to dissolve completely to yield a clear solution. A glass plate $(15 \times 15 \mathrm{~cm})$ thoroughly cleaned with hot water and then with acetone was used as a substrate.

To achieve perfect levelling and uniformity in the thickness of the film, a pool of mercury was used in a plastic tray in which the glass plate was freely suspended. The solution was poured on the glass plate and allowed to spread uniformly in all directions on the substrate. The whole assembly was placed in a dust free chamber maintained at a constant temperature $\left(40^{\circ} \mathrm{C}\right)$. In this way, the film was prepared by isothermal evaporation technique (Bahri and Sood 1983; Mehendri et al 1996). The film was subjected to $12 \mathrm{~h}$ heating at a constant temperature of $50^{\circ} \mathrm{C}$ and for another $12 \mathrm{~h}$ at room temperature to remove traces of solvent. Finally, the film was removed from the glass plate. It was cut into small pieces of suitable size, which were washed with ethyl alcohol to remove the surface impurities. The $\mathrm{X}$-ray powder diffractogram of the sample, recorded from a Phillips (Holland) automated diffractometer (RSIC, Nagpur) is shown in figure 1 . The absence of peak in the $\mathrm{X}$ ray spectra confirmed the amorphous nature of the polymer sample.

\subsection{Thickness measurements}

For measuring the thickness, micrometer screw gauge (Mahendri et al 1996) with least count, $0.001 \mathrm{~cm}(10 \mu \mathrm{m})$, was used. But for greater accuracy and resolution, a compound microscope in conjunction with an acculometer which gives least count, $1.3 \mu \mathrm{m}$ and $3.3 \mu \mathrm{m}$, at the magnification of $1: 10$ and $1: 100$, respectively, was used. A small section of the sample was taken and mounted vertically to get a clear view of the thickness. The film used for the present study is of thickness, $60 \mu \mathrm{m}$.

\subsection{Electrode coating}

The electrode coating on the film of measured thickness was done by using quick drying and highly conducting silver paste (Sangawar 1995; Mehendri et al 1996) supplied by Eltecks Corporation, Bangalore. A mask of a circular aperture of $2.5 \mathrm{~cm}$ diameter was used while coating, to ensure uniformity in size of coated silver electrode.

\subsection{Measurements}

The thermostatically controlled oven supplied by the Tempo Industrial Corporation, Mumbai, was used for heating purpose. A mercury thermometer with an accuracy of $\pm 1^{\circ} \mathrm{C}$ was used for recording temperatures. The regulated power supply supplied by Nupur, India, was used as the voltage

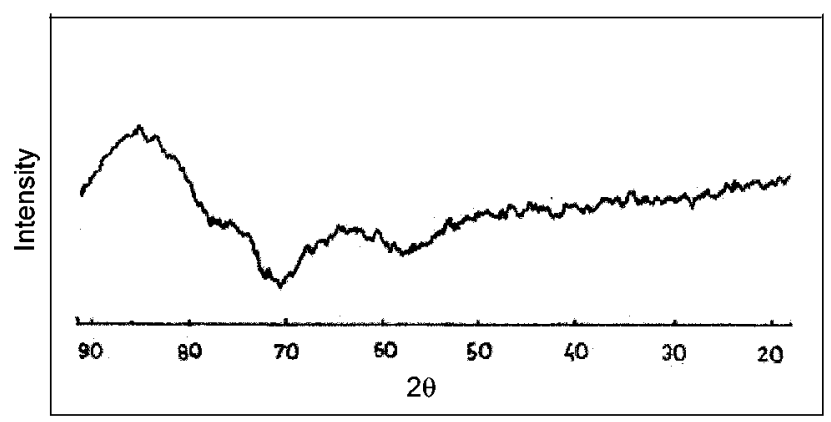

Figure 1. X-ray diffractogram of sample, polyaniline doped PVC-PMMA polymer blends. 
source, while current was recorded by using highly sensitive Pico-Ammeter (Model DPA 111) with an accuracy of $\pm 0 \cdot 2 \%$ supplied by Scientific Equipments, Roorkee.

The sample film with silver electrodes was sandwiched between two brass electrodes (diameter, $2.4 \mathrm{~cm}$ ) of the sample holder specially fabricated in our laboratory. This formed the metal-insulator-metal (M-I-M) system, which was placed in the furnace. The current $(I)$-voltage $(V)$ measurements were done at various temperatures from 323 $363 \mathrm{~K}$.

\section{Results and discussion}

$\operatorname{Ln}(I)$ vs $\ln (V)$ plots of polyaniline doped PVC-PMMA thin films at various temperatures $323 \mathrm{~K}, 333 \mathrm{~K}, 343 \mathrm{~K}, 353 \mathrm{~K}$ and $363 \mathrm{~K}$ are shown in figure 2 . The current increases non-linearly with applied voltage and does not follow a power law, $I=k V^{m}$, where $k$ and $m$ are constants. The possibility of ohmic conductions as well as space charge limited conduction is ruled out from the observed behaviour of $I-V$ characteristics. This is also evident from the fact that Ohm's law follows from the free electron model of a metal. The free electron in a metal undergoes frequent collisions, which are not billiard-ball collisions with other electrons, but represent the scattering of electron waves by irregularities in the crystal structure, both defects such as impurity atoms and also atoms temporarily out of placed as they vibrate (The atom of a perfect crystal lattice do not scatter free electron wave except under certain specific circumstances). In the present case the constituents of blends are itself insulators and blends almost amorphous, giving wide scope for irregularities in the structure and so ruling out ohmic conduction.

Regarding space charge limited conduction, it follows that electrical conduction may occur through the movement of either electrons or ions. The polymeric subgroup falls at low conductivity end. In most polymeric materials it is very difficult to observe any electronic conductivity at all and

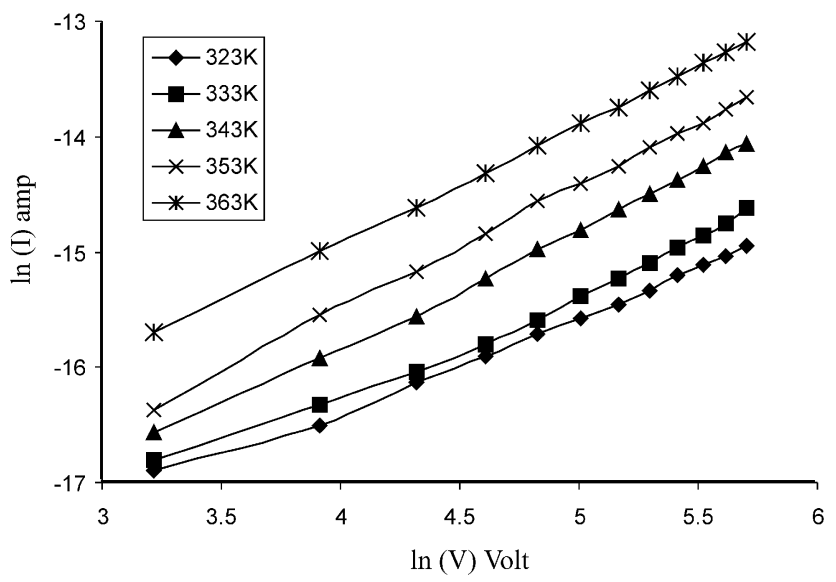

Figure 2. Current-voltage characteristics. what conductivity there is, usually depends upon movement of adventitious ions. Naturally with so feeble a charge carrier density, space charge limited conduction seems a remote possibility.

We now discuss analytically the probable mechanism of conduction.

\subsection{Poole-Frenkel mechanism}

The current-voltage relationship for Poole-Frenkel mechanism is expressed as

$$
J=B \exp \left[\frac{-\varphi}{k T}+\beta_{\mathrm{PF}} E^{1 / 2}\right],
$$

where

$$
\beta_{\mathrm{PF}}=\frac{e}{k T}\left[\frac{e}{\pi \varepsilon \varepsilon_{0} d}\right]^{1 / 2}=\text { constant, }
$$

where $B$ is a constant and all other symbols have their usual meanings. The Poole-Frenkel mechanism predicts a field dependent conductivity as

$$
\begin{aligned}
& \sigma=\sigma_{0} \exp \left[\frac{\beta_{\mathrm{PF}} E^{1 / 2}}{2 k T}\right], \\
& \text { or } \ln \sigma=\ln \sigma+\left(\beta_{\mathrm{PF}} E^{1 / 2} / 2 k T\right),
\end{aligned}
$$

so that the Poole-Frenkel mechanism is characterized by the linearity of $\ln \sigma$ vs $E^{1 / 2}$ plots i.e. Poole-Frenkel plots predicted by (3) are linear with a +ve slope.

In the present case of polyaniline doped polyvinyl chloride (PVC) and poly(methyl methacrylate) (PMMA) thin films, it can be inferred from the Poole-Frenkel plots in figure 3 that this mechanism does not contribute significantly to the conduction as $\ln \sigma$ does not show appreciable dependence on $E^{1 / 2}$ axis, indicating absence of PF mechanism.

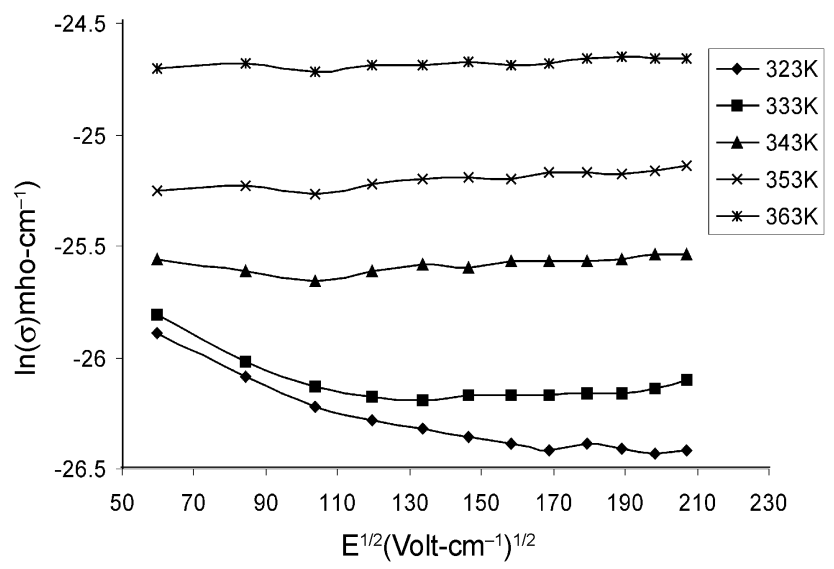

Figure 3. Poole-Frenkel plots. 


\subsection{Fowler-Nordheim mechanism}

The Fowler-Nordheim relation (Fowler 1928) for current density is

$$
J=A V^{2} \exp \left[\frac{-\varphi}{V}\right],
$$

so that,

$$
\ln \left[\frac{J}{V^{2}}\right]=\ln A-\left[\frac{\varphi}{V}\right],
$$

and the $\ln J / V^{2}$ vs $1000 / V$ plots is expected to be a linear straight line relation with a-ve slope.

In the present case, the $\ln \left(J / V^{2}\right)$ vs $1000 / V$ plots for the sample are presented in figure 4 , which are nearly straight lines with a +ve slope for higher as well as lower values of $V$ indicating the absence of tunneling current as suggested by $F-N$ relation.

\subsection{Schottky plots}

The Schottky-Richardson current voltage relationship is expressed as

$$
J=A T^{2} \exp \left[\frac{-\varphi_{\mathrm{s}}}{k T}+\beta_{\mathrm{SR}} E^{1 / 2}\right],
$$

$\beta_{\mathrm{SR}}$ being the field lowering constant given by,

$$
\beta_{\mathrm{SR}}=\frac{e}{k T}\left[\frac{e}{4 \pi \pi \varepsilon_{0} d}\right]^{1 / 2},
$$

and hence $\ln J=\ln A T^{2}-\varphi_{\mathrm{S}} / k T+\beta_{\mathrm{SR}} E^{1 / 2}$ and that $\ln J$ vs $E^{1 / 2}$ plots should be a straight line with a +ve slope. The results plotted with axes marked in this way are referred to as Schottky plots and linear +ve slope on Schottky plots generally characterize Schottky-Richardson mechanism. Schottky plots for the case (figure 5) are straight lines

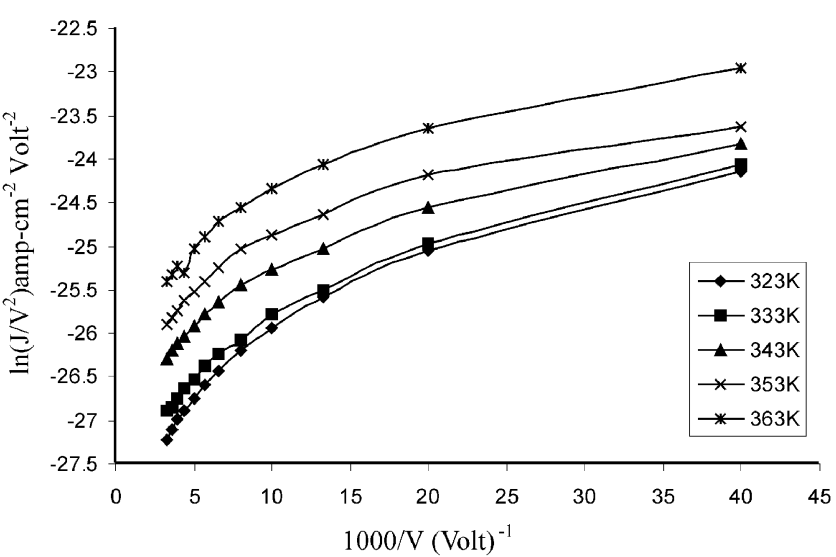

Figure 4. Fowler-Nordheim plots. with positive slope indicating the applicability of the mechanism. Further, in the case of Schottky-Richardson mechanism the current shows strong temperature dependence but not in case of the Poole-Frenkel mechanism. The study of temperature dependence of current density is, therefore, of great importance.

\subsection{Current density vs temperature plots}

The temperature dependence of current density is presented in the form of $\ln (J)$ vs temperature plots (figure 6 ) which shows that $\ln (J)$ increases linearly with temperature. The strong temperature dependence is in agreement with the Schottky-Richardson mechanism. Further, the straight line with constant slope is observed for all the fields indicating the absence of any thermodynamic transition in the temperature range studied.

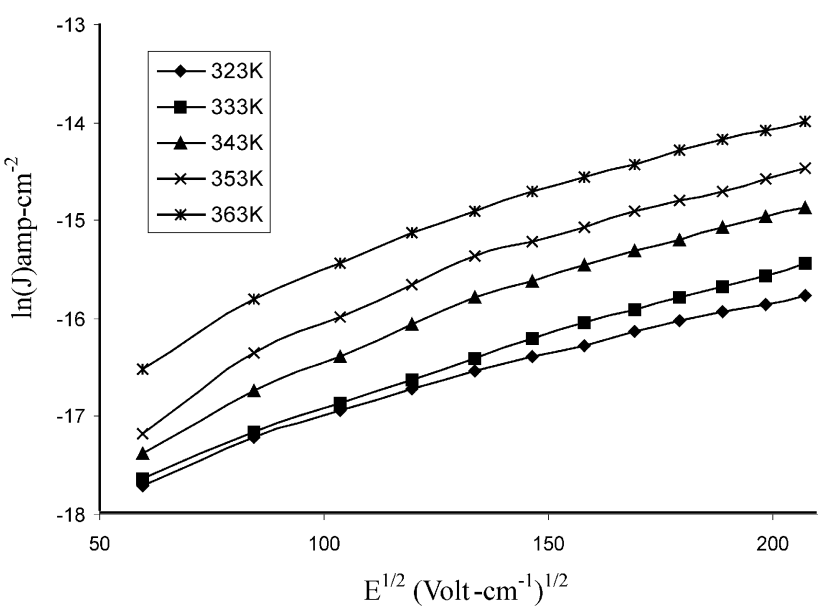

Figure 5. Schottky plots.

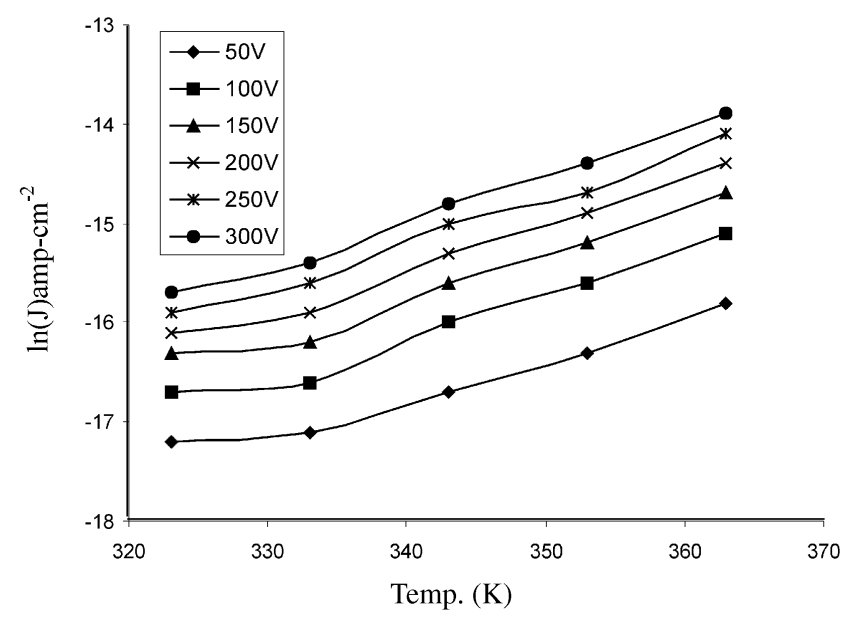

Figure 6. Current density vs temperature plots. 


\subsection{Richardson mechanism}

The Richardson current-voltage relation is expressed as

$$
\begin{aligned}
& J=A T^{2} \exp \left[\frac{-\varphi_{\mathrm{S}}}{k T}+\beta_{\mathrm{SR}} E^{1 / 2}\right], \\
& \frac{J}{T^{2}}=A \exp \left[\frac{-\varphi_{\mathrm{S}}}{k T}+\beta_{\mathrm{SR}} E^{1 / 2}\right], \\
& \ln \frac{J}{T^{2}}=\ln A+\left[\frac{-\varphi_{\mathrm{S}}}{k T}+\beta_{\mathrm{SR}} E^{1 / 2}\right], \\
& \ln \frac{J}{T^{2}}=\ln A+\beta_{\mathrm{SR}} E^{1 / 2}-\frac{\phi_{\mathrm{S}}}{k T} .
\end{aligned}
$$

The graph between $\ln \left(J / T^{2}\right)$ vs $(1 / k T)$ from this relation should be a straight line with a -ve slope. These plots are referred to as Richardson plots and Schottky-Richardson mechanism requires linearity of these plots. In the present case such straight line graphs have been obtained with a -ve slope. The linearity of the plots support Schottky-Richardson mechanism (figure 7).

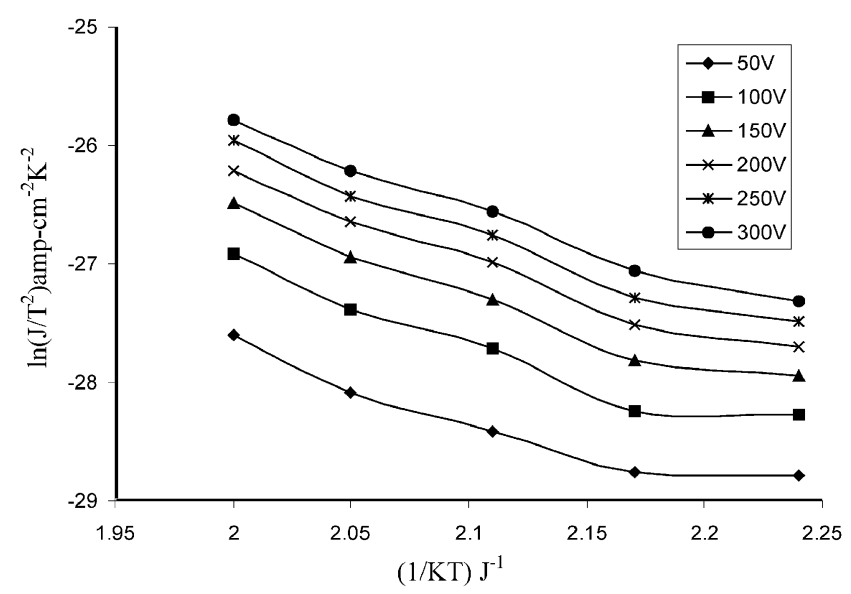

Figure 7. Richardson plots.

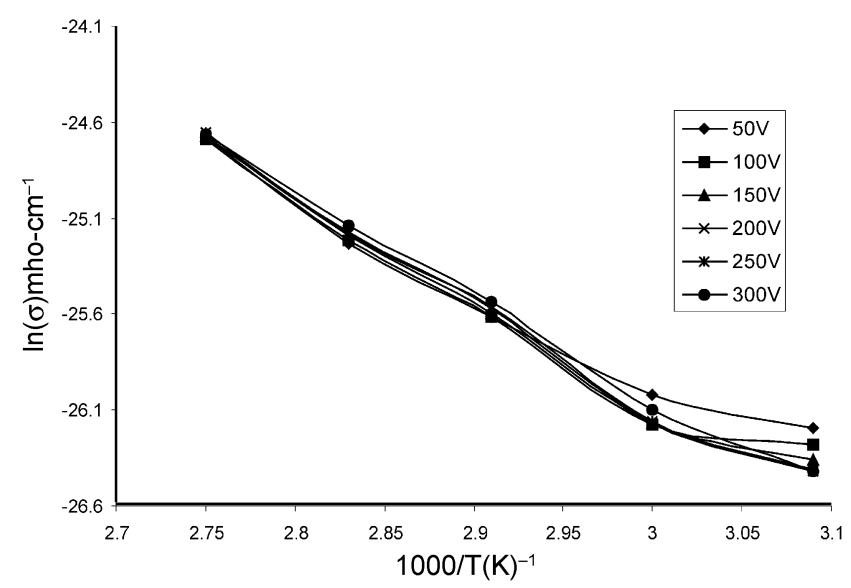

Figure 8. Arrhenius plots.
In case of PF mechanism, the current is due to the thermal excitation of trapped electrons via field assisted lowering of trap depth. While in Schottky model it is due to the thermal activation of electrons over metal-insulator interface barrier with added effect of applied field reducing barrier height. Also in former electrons are liberated from traps while in the latter electron emission takes place from cathode. Further no strong temperature dependence current is observed in the former while the latter shows a strong temperature dependence.

FN mechanism characterizes tunneling currents. In the light of the comparative characteristic features of different mechanisms of conduction and the observed behaviour of the sample, it can be safely concluded that it is the SchottkyRichardson mechanism that is operating in the present case.

\subsection{Arrhenius plots}

The temperature dependence of conductivity of polyaniline doped PVC-PMMA thin film is presented in the form of Arrhenius plots (figure 8$)$ i.e. $\ln (\sigma)$ vs $1000 / T$ plots at all values of applied voltage, showing a straight line with a -ve slope. From the slope of the straight line, the activation energy is calculated and found to be in the neighbourhood of $0.51 \mathrm{eV}$. This is in good agreement with the reported order of magnitudes.

\section{Conclusions}

After studying electrical conduction through doped sample film under various existing mechanisms, it is observed that in the present case, the behaviour cannot be described by $\mathrm{P}-\mathrm{F}$ and $\mathrm{F}-\mathrm{N}$ mechanisms but can be closely described by Schottky and Richardson mechanisms. This is also evident from Arrhenius plots, which give activation energy of $0.51 \mathrm{eV}$, which lies in the semiconducting range. This is the case with the present sample, polyaniline doped PVC-PMMA.

\section{Acknowledgement}

The authors are thankful to Prof. V G Bhamburkar, Principal, Shri Shivaji Science College, Amravati, for providing necessary laboratory facilities during the course of this work.

\section{References}

Awasthi S K and Bajpai R 2001 Indian J. Pure \& Appl. Phys. 397793

Bahri R and Sood B R 1983 Thin Solid Films 100 L5

Belsare N G and Deogaonkar V S 1998 Indian J. Pure \& Appl. Phys. 36280 
Bhat N V and Makwana D N 1990 Indian J. Pure \& Appl. Phys. 28460

Burghate D K, Deshmukh S H, Laxmi Joshi, Deogaonkar V S and Deshmukh P T 2004 Indian J. Pure \& Appl. Phys. 42533

Chakraborty S C, Patil N B, Das S K and Basu S 1991 Indian J. Pure \& Appl. Phys. 29478

Danno T, Miyasaka K and Ishikawa K 1983 J. Polym. Sci. Polym. Phys. Ed (USA) 211527

Deshmukh S H, Burghate D K, Deogaonkar V S and Deshmukh P T 2005 Indian J. Phys. 791263

Ferraro J R and Walkar A 1965 J. Chem. Phys. 421273

Frenkel J 1938 Phys. Rev. 54647

Fowler R H and Nordheim L 1928 Proc. R. Soc. London A119 173

Karimi N A, Gupta D and Prasad P 1987 Indian J. Pure. \& Appl. Phys. 32160

Keton J E (ed.) 1968 Organic semiconducting polymers (New York: Marcel Dekker) p. 267

Keller J M, Dubey S and Datt S C 1991 Indian J. Pure \& Appl. Phys. 29150

Khare P K 1994 Indian J. Pure \& Appl. Phys. 32160
Khare P K and Shriwastava A P 1991 Indian J. Pure \& Appl. Phys. 291410

Kimura T and Kajiwara M 1998 J. Mater. Sci. 332955

Kryezewski M 1975 Polym. Sci. Polym. Symp. (USA) 50359

Kumar N, Malhotra B D and Chanda S 1985 J. Polym. Phys. Ed. (USA) 2357

Mead C A 1961 J. Appl. Phys. USA 33646

Mehendri P C, Agrawal J P and Jain K 1996 Indian J. Pure \& Appl. Phys. 34101

Parak N C and Garg T C 1991 Indian J. Pure \& Appl. Phys. 25 110

Radhakrishnan S 2001 Proceedings of national conference on recent trends in composites 1 (Amravati: Shri Shivaji Science College)

Rose A 1955 Phys. Rev. 791538

Sangawar V S 1995 Study of dielectric and other properties of doped thin film polystyrene (PS) and poly(methyl methacrylate) (PMMA) thermoelectrets, Ph D Thesis, Amravati University, Amravati

Schottky W 1914 Z. Phys. 15872 